\title{
A BROAD IRON LINE IN THE CHANDRA/HETG SPECTRUM OF 4U 1705-44
}

\author{
T. Di Salvo, ${ }^{1}$ R. Iaria, ${ }^{2}$ M. Mćndez, ${ }^{3}$ L. Burderi, ${ }^{4}$ N.R. Robba,${ }^{2}$ L. Stella,${ }^{4}$ and M. van der Klis ${ }^{1}$
}

We present preliminary results from a Chandra $30 \mathrm{ks}$ observation of the atoll source 4U 1705-44. In particular we concentrate on the study of the iron $\mathrm{K} \alpha$ line, using the HEG spectrometer. The iron $\mathrm{K} \alpha$ line at $\sim 6.6 \mathrm{keV}$ is found to be intrinsically broad ( $F W H M \sim 1.7 \mathrm{keV})$; its width can be explained by reflection from a cold accretion disk extending down to $\sim 17 \mathrm{~km}$ from the neutron star center or by Compton broadening in the hotter $(\sim 3-4 \mathrm{keV})$ corona.

Broad emission lines (FWHM up to $\sim 1 \mathrm{keV}$ ) at energies in the range $6.4 \quad 6.7 \mathrm{keV}$ are often observed in the spectra of low-mass X-ray binaries hosting an old accreting neutron star. These lines are identified with the Kir radiative transitions of iron at different ionization states. These features are powerful tools to investigate the structure of the accretion flow close to the central source; in particular, important information can be obtained from the detailed spectroscopy of the iron $\mathrm{K} \alpha$ emission line and absorption edge, since they are determined by the ionization state, geometry and velocity field of the reprocessing plasma. 4U 170544 is an atoll source (see Hasinger \& van der Klis 1989) which also shows type-I X-ray bursts. We selected this source, in which a broad (1.1 keV FWHMI) iron (nission line at $6.5 \mathrm{keV}$ has been reported (White 't al. 1986; Barret \& Olive 2002), for a Chandra observation in order to study the iron line profile and to discriminate among the various models that have been proposed to explain the large line width.

Our $30 \mathrm{ks}$ observation of $4 \mathrm{U} 1705-44$ was performed using the High-Energy Transmission Grating Spectrometer (HETGS) on board of Chandra starting on 2001 July 1. The HETGS consists of two transmission gratings, the Modium-Energy Grating (MEG) and the High-Energy Grating (HEG). It allows high-resolution spectroscopy from 1.2 to $31 \AA$

\footnotetext{
${ }^{1}$ Astronomical Institute "Anton Pannekoek," University of Amsterdam and Center for High-Energy Astrophysics, Kruislaan 403. NL 1098 SJ Amsterdam, the Netherlands.

${ }^{2}$ Dipartimento di Scienze Fisiche ed Astronomiche, Iniversità di Palermo, Italy.

${ }^{3}$ National Institute for Space Research, SRON, Itrecht. the Netherlands.

${ }^{4}$ Osservatorio Astronomico di Roma, Italy.
}

$(0.4-10 \mathrm{keV})$ with a peak spectral resolution at $12 \AA$ of $\Delta \lambda / \lambda \sim 1000$ for HEG first order. In our analysis, we utilize the HEG first-order spectrum, which is less affected by pile-up (less than 10\%) with respect to the MEG first-order spectrum.

We fit the HEG spectrum of $4 \mathrm{U} 1705-44$ to a continuum model. The best fit model consists of a blackbody plus the Comptonization model comptt (Titarchuk 1994), modified by absorption from neutral matter, which gives a $\chi_{\text {red }}^{2}($ d.o.f.) of 1.64(846). The addition of a broad $(\sigma \sim 0.7 \mathrm{keV})$ Gaussian line centered at $6.57 \mathrm{keV}$ proves necessary, giving $\chi_{\text {red }}^{2}($ d.o.f. $)=1.25(843)$. The high energy resolution of the HEG shows that the line is intrinsically broad (FWHM $\sim 1.7 \mathrm{keV}$ ). Therefore the most probable origin of this line is an accretion disk (in this case the large width of the line would be due to Doppler and relativistic smearing effects) or from a hot corona (in this case the large width of the line would be due to Compton broadening).

In the case the line is produced by reflection in an accretion disk we estimate that the required inner radius of the disk is $8 R_{g}$ or $\sim 17 \mathrm{~km}$ for a $1.4 M_{\odot}$ neutron star. The inclination angle is $\sim 80^{\circ}$, which seems to be quite high for this source. Alternatively, Comptonization in the corona could explain the large width of the line. Detailed calculations give $\sigma_{\mathrm{Fe}}=0.019 E_{\mathrm{Fe}} \tau_{T}\left(1+0.78 k T_{e}\right)$, where $\tau_{T}$ is the Thompson optical depth and $k T_{e}$ is in $\mathrm{keV}$ (Kallman \& White 1989, see also Brandt \& Matt 1994). Assuming an electron temperature $k T_{e}=3.8 \mathrm{keV}$, as derived from the fit of our data to the Comptonization model, we can explain the width of the iron line for a Thomson optical depth of $\tau_{T} \sim 1.4$. Therefore, it is possible that the line is produced in the outer region of the Comptonizing corona, where the optical depth decreases (assuming that the temperature remains constant).

\section{REFERENCES}

Barret, D., \& Olive, J.F., 2002, ApJ, 576, 391

Brandt, W.M., \& Matt, G., 1994, MNRAS, 268, 1051

Hasinger, G., \& van der Klis, M., 1989, A\&A, 225, 79

Kallman, T., \& White, N.E., 1989, ApJ, 341, 955

Titarchuk, L., 1994, ApJ, 434, 570

White, N.E., et al., 1986, MNRAS, 218, 129 